\title{
A Necrotized Eyelid
}

\author{
Hideharu Hagiya ${ }^{1}$, Ekkachai Thiansukhon ${ }^{2}$, Yukihiro Akeda ${ }^{1}$ and Kazunori Tomono ${ }^{1}$
}

Key words: endophthalmitis, group-A hemolytic streptococcus, periorbital necrotizing fasciitis, Streptococcus pyogenes, toxic shock syndrome

(Intern Med 55: 2121, 2016)

(DOI: 10.2169/internalmedicine.55.6574)

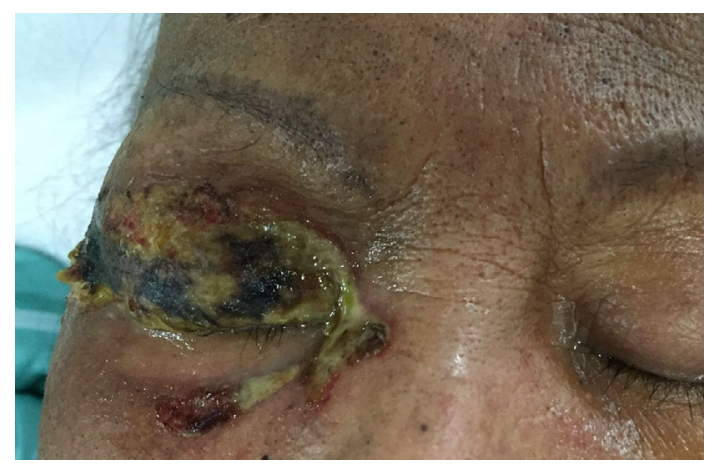

Picture.

A 62-year-old Thai woman administered prednisolone for systemic lupus erythematosus complained of progressive swelling of her right eyelid accompanying a high fever. The patient developed shock, and Streptococcus pyogenes was isolated from her blood and periorbital tissues. Under a diagnosis of periorbital necrotizing fasciitis (PNF) and endophthalmitis complicated with streptococcal toxic shock syndrome, she underwent intensive care and successfully recovered. However, the periorbital lesion remained necrotic (Picture), resulting in poor visual acuity of hand motion.
PNF is extremely rare with an incidence rate of less than 1 per million per year (1). Group A $\beta$-hemolytic Streptococcus is the most common pathogen and complication of toxic shock syndrome is frequently observed $(1,2)$. Although the mortality of PNF is relatively low (approximately 10\%) compared to that occurring in the extremities, the infection can result in blindness and cosmetic issues. Early awareness followed by appropriate treatment is essential for a better prognosis of this infection.

The authors state that they have no Conflict of Interest (COI).

\section{Acknowledgement}

The authors experienced the presenting case during the 7 th Training Course on Tropical Infectious Diseases for Japanese Doctors at Udon Thani Hospital (Thailand), hosted by the Research Institute for Microbial Diseases, Osaka University.

\section{References}

1. Flavahan PW, Cauchi P, Gregory ME, Foot B, Drummond SR. Incidence of periorbital necrotising fasciitis in the Uk population: a BOSU study. Br J Ophthalmol 98: 1177-1180, 2014.

2. Lazzeri D, Lazzeri S, Figus M, et al. Periorbital necrotising fasciitis. Br J Ophthalmol 94: 1577-1585, 2010.

\footnotetext{
${ }^{1}$ Division of Infection Control and Prevention, Osaka University Hospital, Japan and ${ }^{2}$ Department of Internal Medicine, Udon Thani Hospital, Thailand

Received for publication September 15, 2015; Accepted for publication October 18, 2015

Correspondence to Dr. Hideharu Hagiya, highgear@hp-infect.med.osaka-u.ac.jp

(C) 2016 The Japanese Society of Internal Medicine Journal Website: http://www.naika.or.jp/imonline/index.html
} 